\title{
Motor and sensory conduction velocities and amplitude of nerve or muscle potentials in the normal rat according to age
}

\author{
Claudine Hort-Legrand, R. Lestrade, A. Behar
}

Service de biophysique UFR Broussais Hôtel-Dieu, Université Pierre et Marie Curie, Paris VI, France

Received: July 4, 2001

Accepted: November 11, 2001

The relative changes in sensory and motor nerve conductions and SNAP and CMAP amplitudes were studied on the sural and tibial posterior nerves in anesthetized male rats, between the 1st and the 23rd month. Neural growth was controlled with the measure of the nerve path length on the skin, between stimulating and recording cathodes for the sural nerve and proximal and distal stimulating cathodes for the tibial posterior nerve. The sural SCV and SNAP amplitude are consistent with a more accurate method than the H-reflex one.

Similar changes were observed in both parameters. During the maturation of the peripheral nervous system, between the 1st and the 5th month, parameters rapidly increased. Over 14 months old, parameters decrease: the diminution of SNAP and CMAP amplitudes is characteristic of aging. The results were analyzed through quadratic and linear regression and were similar to those in young and elderly human patients. Parabola curves fitted the best way to represent the evolution of parameters. Moreover, the linear regression permitted to divide the rat life in 3 parts and to distinguish a period between the 6th and 13th months during which studied parameters are considered as constant. SCV, MCV, SNAP and CMAP amplitudes from the 1st to the 5th, from 6th to 13th and over the 14th month, could be used as reference.

Keywords: rats, maturation, ageing, sensory and motor nerve conductions, neural growth

Electrophysiology is a good tool for a non-invasive investigation of axonal function. The privileged experimental model for peripheral neuropathies with toxic ( 7 , $9)$, metabolic $(10,17,20)$ or traumatic $(1,2,8,14,23)$ origins is the rat sciatic nerve, in histological or electrophysiological studies.

Correspondence should be addressed to

Claudine Hort-Legrand. M.D, PHD

Service de Biophysique UFR Broussais Hôtel-Dieu

75270 Paris CEDEX 06. France

15 rue de l'Ecole de Médecine

Phone and fax: 33.01.46.33.31.18

E-mail: claudine.hort-legrand@bct.ap-hop-paris.fr 
Some publications focused on the age-related motor conduction velocity, which has sometimes been related to histology. In one-old publication (4), sciatic nerve velocity was studied longitudinally from 50 to 850 days of age. Motor conduction velocity (MCV) increased during development up to about 300 days and did not change significantly thereafter. MCV seems related with maturation process but does not seem to be related with aging. More recently (18) the conduction velocity of the sensory and motor nerves with the H-reflex were determined in rats of different weights from 100 to $500 \mathrm{~g}$ (age 1 to 12 months). Sensory conduction velocity (SCV) was always apprehended by H-reflex which measures nerve conduction orthodromically along proprioceptive 1A afferent nerve fibers. In animals, SCV using a peripheral sensory nerve as sural nerve have not been determined previously. Moreover no study combining the measurements of MCV and NCV in the normal rat has been made so far.

According to the aging process, electrophysiologic studies of the caudal nerve revealed reductions in amplitude and conduction velocity between 20 and 30 months of age (11). In a hybrid strain of rats (26), MCV and H-reflex latency were performed on the tibial nerve from rats aged 6,18 and 30, 32 months. Motor distal latency in the 32 months old group was compared to the 6 and 18 months old group and no statistically significant change of the MCV was found in the old rats, but no control group was used.

The failure of longitudinal results was considered. The aim of this experiment is to establish the values of SCV in the sural nerve and MCV in the posterior tibial nerve and to study the changes of sensory nerve action potential (SNAP) and compound motor action potential (CMAP) amplitudes according to growth and aging during a period going from the 1 st to the 23 rd month of age, in male rats. These results should help for age related pharmacological peripheral neuropathies.

\section{Materials and Methods}

\section{Materials}

Among a group of 28 Wistar male rats (rearing in conformity with ISO 9002 norms), without genetic or general disease: 10 were examined between the 1 st and the 13th month after birth, 10 were examined between the 2 nd and the 15 th month and 8 were examined between the 1st and the 23rd month; 3 from this 3rd group died between the 16th and 17th months and the 5 others were examined beyond the 18th month till 23rd months. The average lifetime was $26 \pm 4$ months.

For each test, anesthesia was induced through an intraperitoneal injection of pentobarbital $(40 \mathrm{mg} / \mathrm{kg})$ and sustained with an $\mathrm{O}_{2} /$ fluothane mix. The animal was placed on a homeothermic operating table. A temperature-sensing probe, inserted 
rectally, permitted to maintain the temperature constant $\left(37.5 \pm 0.5^{\circ} \mathrm{C}\right)$ during investigations. Then temperature was checked during the spontaneous awakening. During the study, animals were statistically randomly chosen from each group but no animal was ever anesthetized more than twice a month.

\section{Measurement of motor velocity}

For the measurement of the MCV of the posterior tibial nerve, at the proximal point of stimulation, an $1 \mathrm{~ms}$ square wave pulse was delivered through a $0.5 \mathrm{~mm}$ diameter, $20 \mathrm{~mm}$-length pair of monopolar recording needle electrodes. The cathode was inserted in the sciatic notch and positioned close to the sciatic nerve. The reference electrode was inserted at $10 \mathrm{~mm}$ from the cathode. At the distal point of stimulation, $5 \mathrm{~mm}$-length and $0.5 \mathrm{~mm}$-diameter recording electrodes were subcutaneous, the cathode was inserted behind the internal malleolus and the anode was inserted behind the external one. A needle ground electrode was placed between the 2 points of stimulation. Output impedance was less than $1 \mathrm{k} \Omega$. The muscle potential was recorded by bared steel tip subcutaneous electrodes. They were positioned in the first interosseous space. With $12 \pm 1 \mathrm{~mA}$ of current pulses, motor potential amplitudes were maximal. The filter bandpass was $50-2000 \mathrm{~Hz}$.

The limb was extended fully so as to measure nerve path length between stimulating cathodes on the skin. Latency difference of the onset of the motor potentials was used for the MCV calculation.

The amplitude between the onset and the peak of the muscle potential was also measured (CMAP amplitude). Beyond the 16th month, the morphology of the motor action potential changed and was characterized by compound motor action potential dispersion. The amplitude of the highest compound was measured.

\section{Measurement of sensory velocity}

For the measurement of the SCV, the sural nerve was stimulated under the external malleolus with the small electrodes previously described. Two mm separated subcutaneous cathode and anode. The ground electrode was placed as before. The nerve potential was recorded in the sciatic notch, by the same near-nerve electrodes used for the motor proximal stimulation. The nerve was stimulated at $1.72 \mathrm{~Hz}$ and $12 \mathrm{~mA} .100$ responses were averaged by a computer that rejected the artefacts. The distance between stimulating and recording cathodes was measured on the skin and used for the calculation of the conduction velocity. 


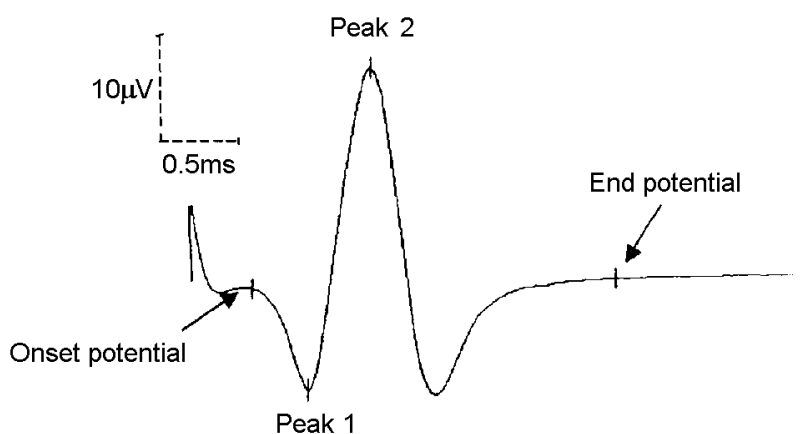

Fig. 1. Sensory potential of sural nerve as recorded in the sciatic notch (triphasic form)

The latency of the first positive peak nerve action potential (peak 1) was measured (Fig. 1). This measure is much more precise than the measure recorded at the onset of the potential and only induces a low error on the conduction velocity of the fastest fibers (6). The amplitude between the first positive phase and the next negative one was measured (SNAP amplitude).

The surface of the sensory potential was automatically computed between the onset and the end of the nerve potential (Fig. 1).

The obtained data were captured and processed statistically through SPSS software (21). The data were compared with an ANOVA test followed by a post hoc Bonferroni test.

\section{Results}

On the 1 st month, the lowest weight was $205 \mathrm{~g}$. The average weight was $427 \pm$ $4.5 \mathrm{~g}$ on the $3 \mathrm{rd}$ month, $558 \pm 8.9 \mathrm{~g}$ on the 5 th month, $696 \pm 22 \mathrm{~g}$ on the 12 th month and remained more or less constant $(760 \pm 58 \mathrm{~g})$ on the 18 th month and later.

\section{Motor parameters}

With the quadratic regression $\left(y=\right.$ a.age $\left.{ }^{2}+b . a g e+c\right)$, curves of all data of the conduction velocity, proximal and distal amplitudes are represented by parabolas. The equations of the more detailed analysis are given in Table I and the corresponding curves are plotted in Figures 2 and 3. 
Table I

Quadratic regression analysis of nerve conduction velocities and of motor and sensory amplitudes

\begin{tabular}{lllll}
\hline Nerve & \multicolumn{2}{c}{ Parameters } & Equations of the parabolas & $\mathrm{R}^{2}$ \\
\hline Tibial post. & NCV & & $-0.11 \mathrm{x}^{2}+2.963 \mathrm{x}+32.357$ & 0.90 \\
& Amplitude & distal & $-0.0801 \mathrm{x}^{2}+1.562 \mathrm{x}+7.931$ & 0.86 \\
& & proximal & $-0.074 \mathrm{x}^{2}+1.481 \mathrm{x}+5.97$ & 0.83 \\
Sural nerve & NCV & peak 1 & $-0.11 \mathrm{x}^{2}+2.879 \mathrm{x}+44.859$ & 0.81 \\
& & peak 2 & $-0.088 \mathrm{x}^{2}+2.488 \mathrm{x}+28.62$ & 0.88 \\
& Amplitude & & $-0.1 \mathrm{x}^{2}+2.91 \mathrm{x}+5.89$ & 0.88 \\
\hline
\end{tabular}

Change of conduction velocity with age

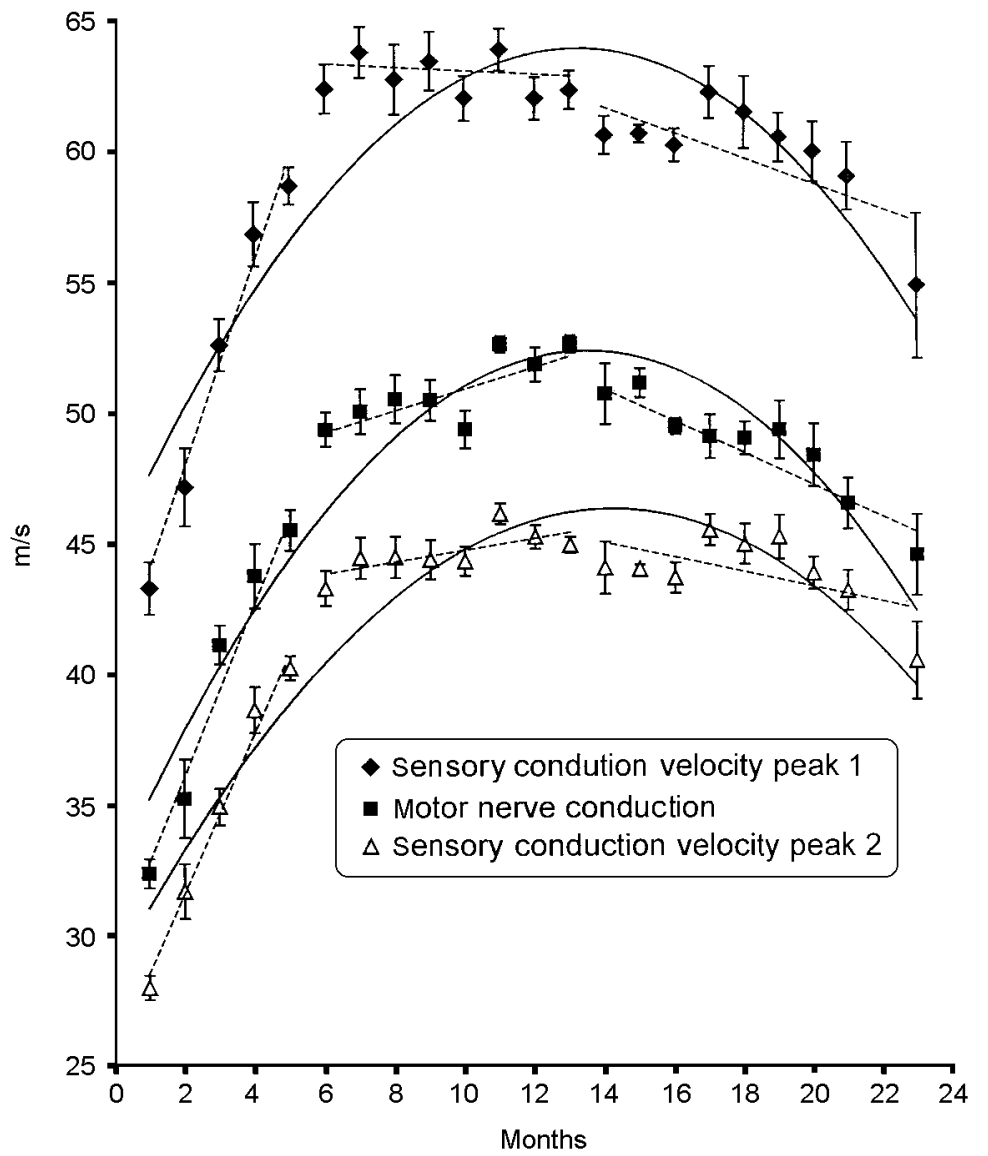

Fig. 2. Curves of the more significant equations (quadratic and linear) of the regression analysis of age and sensory and motor conduction velocities 


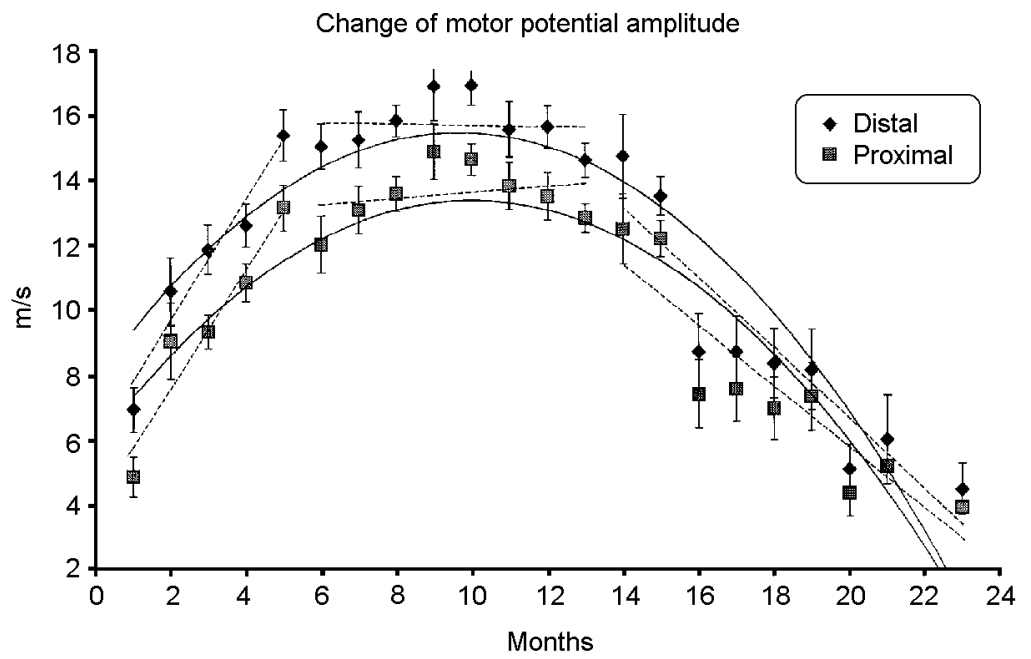

Fig. 3. Quadratic or linear regression analysis of age and distal and proximal motor amplitudes

The motor nerve conductions measured during the first month were clearly inferior to those measured during the $23 \mathrm{rd}$ month $(32.4$ and $44.5 \mathrm{~m} / \mathrm{s}$ respectively, $\mathrm{p}=0$ ). The maximal velocity was reached during the 13 th month $52.6 \pm 0.33 \mathrm{~m} / \mathrm{s}$.

At first, the curves of distal and proximal CMAP amplitudes are separated, then, after the 18th month, they draw closer to each other. The age at which the maxima are reached was 9th month. The minima amplitudes were pretty similar at the beginning and at the end of the experiment $(7 \pm 0.7$ and $4.5 \pm 0.8 \mathrm{mV}, \mathrm{p}=0.05)$ for distal amplitudes and still closer $(4.9 \pm 0.6$ and $3.9 \pm 0.6 \mathrm{mV}, \mathrm{p}=0.30)$, for proximal amplitudes measured at the 1 st and the $23 \mathrm{rd}$ month.

With the linear analysis of the data $(y=\mathrm{a} . \mathrm{age}+\mathrm{b})$ (Table II), MCV rapidly increased between the $1 \mathrm{st}$ and 5th months, from $32.4 \pm 0.6$ to $45.5 \pm 0.7 \mathrm{~m} / \mathrm{s}(\mathrm{p}=0.001)$. MCV kept on increasing from $49.3 \pm 0.6$ to $52.6 \pm 0.33 \mathrm{~m} / \mathrm{s}$ between the 6 th and 13 th months but no significantly $(\mathrm{p}=1)$ then it decreased between the 14 th and the $23 \mathrm{rd}$ month from $51.1 \pm 0.55$ to $44.5 \pm 1.54 \mathrm{~m} / \mathrm{s}(\mathrm{p}=0.008)$.

Distal CMAP amplitude rapidly increased between the 1st and 5th months from $6.9 \pm 0.7$ to $15.4 \pm 0.7 \mathrm{mV}(\mathrm{p}=0.001)$, then remained more or less constant. The average amplitude was $15 \pm 0.7 \mathrm{mV}$ between the 6th and 13th months $(\mathrm{p}=1)$. Then, it decreased after the 14th month. The amplitude was $13.5 \pm 0.62 \mathrm{mV}$ at the 15 th month and $4.5 \pm 0.79 \mathrm{mV}$ at the $23 \mathrm{rd}$ month $(\mathrm{p}=0.001)$. 


\section{Table II}

Linear regression analysis of nerve conduction velocities and motor and sensory amplitudes

\begin{tabular}{|c|c|c|c|c|c|c|c|}
\hline \multirow[t]{4}{*}{ Nerve } & Parameters & \multicolumn{2}{|c|}{$\begin{array}{r}1 \text { to } 5 \text { months } \\
\qquad \mathrm{R}^{2}\end{array}$} & \multicolumn{2}{|c|}{$\begin{array}{r}6 \text { to13 months } \\
\qquad \mathrm{R}^{2}\end{array}$} & \multicolumn{2}{|c|}{$\begin{array}{r}14 \text { to } 23 \text { months } \\
\mathrm{R}^{2}\end{array}$} \\
\hline & $\mathrm{NCV}$ & $3.47 x+29.17$ & 0.96 & $0.44 x+46.67$ & 0.65 & $-0.63 x+60$ & 0.83 \\
\hline & Amplitude distal & $1.89 x+5.8$ & 0.94 & $0.54 x+11.66$ & 0.9 & $-1.1 x+28.49$ & 0.84 \\
\hline & proximal & $1.84 x+3.96$ & 0.92 & $0.7 x+8.01$ & 0.9 & $-0.95 x+24.66$ & 0.82 \\
\hline \multirow[t]{2}{*}{ Sural nerve } & SCV & $4.04 x+39.58$ & 0.98 & $-0.09 x+63.65$ & 0.08 & $-0.52 x+69.35$ & 0.51 \\
\hline & Amplitude & $1.97 x+7.01$ & 0.89 & $0.46 x+18.9$ & 0.26 & $-1.2 x+43.47$ & 0.82 \\
\hline
\end{tabular}

The curve of proximal amplitude seemed to be parallel to the distal curve between the 1st and 23rd months. Curves were shown not to have different slopes between the 1st and 5th $(p=0.99)$ then 6th and 13th $(p=0.90)$ months. They were not separated more significantly between 15 th and $23 \mathrm{rd}$ months $(p=0.99)$. At the $23 \mathrm{rd}$ month, the distal and proximal amplitudes were comparable and equal to $4.5 \pm 0.79$ and $3.9 \pm 0.63 \mathrm{mV}$, respectively (Fig. 3).

\section{Sensory parameters}

Qualitatively, the morphology of the nerve potential did not undergo any modification during the experiment.

With the quadratic regression, the effect of age on peak 1 and peak 2 sural nerve conduction velocities and amplitude of the sensory potential is represented by parabolas (Table I, Fig. 2 and Fig. 4). The maximal conduction velocities and amplitude of the nerve potential were reached around the 13th month. Peak 1 conduction velocity or amplitude of the nerve potential was the lowest at one month $(43.3 \pm 1 \mathrm{~m} / \mathrm{s}$ and $8 \pm 1.1 \mu \mathrm{V}$, respectively) comparatively to the measures taken at the $23 \mathrm{rd}$ month $(54.8 \pm 5.4 \mathrm{~m} / \mathrm{s}$ and $16.8 \pm 2.6 \mu \mathrm{V})$.

Peak 1 and peak 2 velocities curves were statistically parallel but occupied separate positions on the $y$ axis.

With the linear analysis of the sensory parameters (Table II), SCV of peak 1 rapidly increased between the $1 \mathrm{st}$ and the 5 th month, from $43.3 \pm 1$ to $58.7 \pm 0.7 \mathrm{~m} / \mathrm{s}$ $(\mathrm{p}=0.001)$. It slightly decreased between the 6th and 13th months and then significantly decreased between the 14th and 23rd months from $59.6 \pm 1.34$ to $54.8 \pm 2.8 \mathrm{~m} / \mathrm{s}$ $(\mathrm{p}=0.03)$.

SCV of peak 2 follows the same distribution (Fig. 2). 


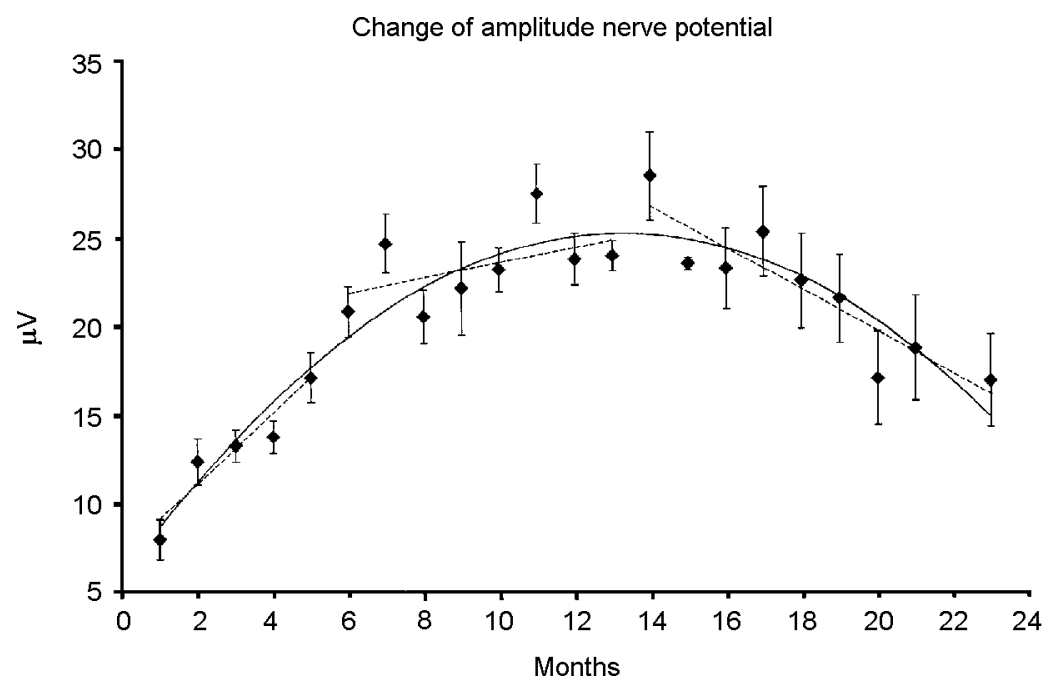

Fig. 4. Quadratic or linear regression analysis of age and sensory potential amplitude

The SNAP amplitude increased from $8.0 \pm 1.1$ to $17.1 \pm 1.4 \mu \mathrm{V}$ between the 1 st and 5th months ( $\mathrm{p}=0.001)$, and then from $20.8 \pm 1.4$ to $23.9 \pm 0.9 \mu \mathrm{V}(\mathrm{p}=1)$ between the 6 th and 13th months. It decreased between the 14th and 23rd months to reach the value of $16.8 \pm 2.6 \mu \mathrm{V}$ (Fig. 4).

The area of the sensory potential remained constant between the 2nd and 23rd months $(7.96 \pm 0.94 \mu \mathrm{V} / \mathrm{ms}$ and $10.2 \pm 1.57 \mu \mathrm{V} / \mathrm{ms}$, respectively, $\mathrm{p}=0.27)$.

\section{Discussion}

Sensory conduction velocity of the sural nerve, motor conduction velocity of the tibial posterior nerve and amplitudes of the nerve and muscle potentials had been longitudinally studied in 28 male rats from their 2 nd to their 13 th month. Five rats were studied till the $23 \mathrm{rd}$ month. It showed a close relationship with age.

The originality of the measurement of SCV lies in the use of a peripheral sensory nerve (sural nerve) whereas the H-reflex was usually recorded as SCV from a mixed nerve, in the animal (18). First, the H reflex raises the question of its nature in the rat: all electrophysiologists do not acknowledge the sensory origin of the late response since this response may persist, though it should disappear, with high stimulation intensities. Second, the technique used here measures a response on a sensory nerve trunk after a distal stimulation, as it is usually practised in human electrophysiology. However, it has been shown that, in the rat, some motor axons use the external sural nerve on their way through the leg and leave it above the ankle to reach the lateral plantar nerve (16). Two 
arguments reinforce our hypothesis that the measure made is the measure of SCV: the care given to the placement of the stimulating electrodes under the external malleolus, that is a priori under the onset of the anastomotic motor axon, and the fact that the sural $\mathrm{NCV}$ is always greater than the tibial posterior MCV. Sural NCV is consistent with interoceptive, IIa or IIb nervous fiber velocities.

Intoxication with different drugs demonstrates the increased sensitivity of the sural NCV measurement. In experimental acrylamide neuropathy (9) both large sensory and motor diameter axons are affected: the H-reflex scores sensory attack. In rats with streptozotocine-induced diabetes, the primary sensory attack concerns small diameter axons (10). The sural NCV is likely to be more sensitive than the H-reflex to explore sensory velocity. Our technique may allow to differentiate conduction velocities according to the diameter of axons: the velocity calculated from peak 1 corresponds to the largest diameter axons, whereas the velocity calculated from peak 2 corresponds to the largest diameter axons mixed with large ones (25) and may be of great interest in this pathology.

Looking at the diagram of the collected data, two forms of regression are used.

First, a quadratic regression analysis $\left(y=a \cdot a g e^{2}+b \cdot a g e+c\right)$. The different parameters develop in an approximately parallel manner, with a maximum at 9 months for distal and proximal tibial posterior CMAP amplitudes and 13 months for sensory and motor conduction velocities as well as for sural SNAP amplitude. These results are consistent with previous investigations in human patients that found a parabolic agerelated CMAP and SNAP amplitudes and motor and sensory velocities, between 20 and 80 years old by Taylor (19).

Second, the simple linear regression $(y=a . a g e+b)$ allows for the division of the development of the parameters in 3 stages: from the 1st to the 5th month, from the 6th to the 13th month and from the 14th to the $23 \mathrm{rd}$ month. The parameters increase very rapidly at first. During the intermediary period, the parameters develop either increasingly or decreasingly, with a slight incline on the representative line. All the parameters decrease between the 14th and the 23rd month.

In humans the maturation of motor and sensory ( $\mathrm{H}$ reflex) nerve conduction velocities of the sciatic nerve develops in an exponential manner between birth and 5 years old. At this age, it reaches an asymptote corresponding to the velocity of an adult (24). An author indicates that adult conduction values are reached around 8 years old (6). Sensory conduction velocities of the median, radial and sural nerves linearly decrease from 15 up to 90 years, for other authors $(12,13,22)$. Clinical practicians usually consider that standard data are constant between 5 and 65 years.

In old rats, the comparison of the linear regression gradients of the parameters between the 14th and 23rd months shows that amplitudes decrease faster than nerve 
conduction velocity (Tables I and II). Progressive and related changes with age in the peripheral nervous system involve both sensory and motor pathways.

In elderly humans, amplitude has a high inter individual variability. However, all the authors describe clearly decreasing amplitude with age from the 50 years old. It depends on the nature of the nerve (sensory or motor) and its location (5). Our study confirms the importance of these decreases in old rats. CMAP amplitude decreases earlier than SNAP one (16 months versus 19 months), this could be explained by a weakness and atrophy of the aging skeletal muscle of the posterior limbs (23).

The decreased nerve conduction could be related to neuronal loss, myelinisation (segmental demyelinisation, early remyelinisation, change in inter-nodal length, thick myelinated fiber) or change in axonal membrane (1). Moreover a relative inactivity of these sedentary rats might account for the decline of all parameters in old rats. This hypothesis is supported by the preventive effect of the exercise on the apparition of the diabetic neuropathy in human (3). However, an age-related neuropathy of the phrenic nerve was described in rats over 23 months old. This peripheral neuropathy is not a pressure neuropathy (15).

In conclusion, this study permits the use of the measurements of sensory and motor velocities on Wistar rats and their interpretation in the course of time. The results highlight the major role of maturation for the peripheral nervous system between the $1 \mathrm{st}$ and the 5th month. The decrease of amplitude of sensory and motor potentials seems to be the main indicator of ageing.

\section{REFERENCES}

1. Adinolfi AM, Yamuy J, Morales FR, Chase MH: Segmental demyelination in peripheral nerves of old cats. Neurobiol. Aging 12, 175-179 (1991)

2. Aldgskogius H, Molander C, Persson J, Thomander L: Specific and non-specific regeneration of motor axons after sciatic nerve injury and repair in the rat. J. Neurol. Sci. 80, 249-257 (1987)

3. Belmin J, Valensi P: Diabetic neuropathy in Elderly Patients. Drugs and Aging 6, 416-429 (1996)

4. Birren JE, Wall PD: Age change in conduction velocity, refractory period, number of fibers, connective tissue space and blood vessels in sciatic nerve of rats. J. Comp. Neurol. 104, 1-16 (1956)

5. Bouche P, Cattelin F, Saint JO: Clinical and electrophysiological study of the peripheral nervous system in the elderly. J. Neurol. 240, 263-268 (1993)

6. Buchtal F, Rosenfalck A: Evoked action potentials and conduction velocity in human sensory nerve. Brain Res. 3, 1-122 (1966)

7. De Koning P, Neut JP, Jennekens FGI, Gispen WH: Evaluation of cis-diamminedichloroplatinum (II) 5 cisplatin neurotoxicity in rats. Toxicol. Appl. Pharmacol. 89, 81-87 (1987)

8. Froidart-Dessale M, Dubuisson A: Sciatic nerve regeneration through venous or nervous grafts in the rat. Exp. Neurol. 148, 236-246 (1997)

9. Fullerton PM, Barnes JM: Peripheral neuropathy in rats produced by acrylamide. Brit. J. Industr. Med. 23, 210-221 (1966) 
10. Jakobsen J: Axonal dwindling in early experimental diabetes. 1. A study of cross sectioned nerves. Diabetologia 12, 539-546 (1976)

11. Knox CA, Kokmen E, Dyck PJ: Morphometric alteration of rat myelinated fibers with aging. J Neuropathol. Exp. Neurol. 48 119-139 (1989)

12. La Fratta CW, Canestrari RE: A comparison of sensory and motor nerve conduction velocities as related to age. Arch. Phys. Med. Rehabil. 47, 286-290 (1966)

13. La Fratta CW, Zalis AW: Age effects on sural nerve conduction velocity. Arch. Phys. Med. Rehabil. 54, 475-477 (1973)

14. Lewin-Kowalik J, Gorka D, Larysz-Brysz M, Goka B, Swiech-Sabuda E, Malecka-Tendera E, Krause M: Short-time predegenerated peripheral nerve grafts promote regrowth of injured hippocampal neurites. Acta Physiol. Hung. 85/3, 259-268 (1998)

15. Kiyokazu O, Koji M, Minoru T, Isao N: Peripheral neuropathy in the spontaneously diabetic WBN/Kob rat. Acta Neuropathol. 92, 603-607 (1966)

16. Peyronnard JM, Charron L: Motor and sensory neurons of the rat sural nerve: a horseradish peroxidase study. Muscle and nerve 5, 654-660 (1982)

17. Sharma AK, Thomas PK: Peripheral nerve structure and function in experimental diabetes. J. Neurol. Sci. 23, 1-15 (1974)

18. Stanley ES: Sensory and motor nerve conduction velocities and the latency of the $\mathrm{H}$ reflex during growth of the rat. Exp. Neurol. 71, 497-506 (1981)

19. Taylor PK: Non-linear effects of age on nerve conduction in adults. J. Neurol. Sci. 66, 223-234 (1984)

20. Thomas PK, Fraher JP, Leary DO, Moran MA, Cole M, King RHM: Relative growth and maturation of axons size and myelin thickness in the tibial nerve of rat. Acta Neuropathol. 79, 375-386 (1990)

21. SPSS Base 8.0 Application guide SPSS Inc. (1998)

22. Vandendriessche G, Vanhecke J, Rosselle N: Normal sensory conduction in the distal segment of the median and radial nerve: relation to age. Electromyogr. Clin. Neurophysiol. 21, 511-519 (1981)

23. Van Meeteren NL, Brakkee JH, Hamers FP, Helders PJ, Gispen WH: Exercise training improves functional recovery and motor nerve conduction velocity after sciatic nerve crush lesion in the rat. Arch. Phys. Med. Rehabil. 78, 70-77 (1997)

24. Vecchierini-Blineau MF, Guiheneuc P: Maturation des vitesses de conduction nerveuse de la naissance à cinq ans. Arch. franç. Pédiat. 36, 563-572 (1979)

25. de Weerd JPC (1984): Volume conduction and electromyography. Current practice of clinical electromyography, ed. Notermans SLH, Elsevier, pp. 9-28.

26. Wineinger MA, Sharman RB, Stevenson TR, Carlsen RC, Mac Donald: Peripheral nerve and muscle function in the aging Fischer 344/brown-norway rat RB. Growth, development \& aging 59, 107-119 (1995) 